\title{
Principales conflictos entre la política de vivienda sustentable y las metas de transición energética en México. El caso del programa Hipoteca Verde \\ Main conflicts between sustainable housing policy and energy transition goals in México. The case of the green mortgage program
}

\begin{abstract}
Catalina Borbolla-Gaxiola* David Carlos Ávila-Ramírez*

Recibido: mayo 15 de 2020.

Aceptado: septiembre 08 de 2020.

Resumen

El presente artículo tiene como objetivo abrir la discusión acerca de las acciones de política de vivienda sustentable en México y las metas planteadas para la transición energética en el país. Para esto, se desarrolla el concepto de transición energética a través de un análisis de la literatura académica, exponiendo además el actual debate teórico en torno a este. Se continúa con una revisión de la política Hipoteca Verde de vivienda sustentable existente en el país, haciendo énfasis en el programa. Finalmente, se exponen tres conflictos principales que muestran la incompatibilidad entre la política de vivienda sustentable, el programa Hipoteca Verde y la transición energética planteada en México, lo cual a largo plazo podría ocasionar que no se cumplan las metas planteadas en materia energética para los próximos años.
\end{abstract}

Palabras clave: transición energética, política de vivienda, energía renovable, generación distribuida, Hipoteca Verde.

\begin{abstract}
This article aims to open the discussion about sustainable housing policy actions in Mexico and the goals set for the energy transition in the country. To achieve this, the concept of the energy transition will be developed through an analysis of academic literature, also exposing the current theoretical debate around it. After that, will be done a review of the existing sustainable housing policy in the country, emphasizing the green mortgage program. It ends with exposing three main conflicts that show the incompatibility between the sustainable housing policy, the green mortgage program, and the energy transition proposal in Mexico, which in the long term would fail to meet the goals set for energy in the coming years.
\end{abstract}

Keywords: Energy transition, housing policy, renewable energy, distributed generation, green mortgage. 


\section{Introducción}

La transición energética es un tema de debate actual en el que México ha participado con la producción de leyes, normas y programas que buscan contribuir en alcanzar las metas planteadas sobre la disminución del uso de energía proveniente de combustibles fósiles, el incremento de energías renovables en el sector eléctrico nacional y la disminución de emisiones contaminantes. Entre estas metas destacan lo establecido en la Ley para el Aprovechamiento de Energías Renovables y el Financiamiento de la Transición Energética, donde se menciona que la Secretaría de Energía fija como meta una participación máxima de $65 \%$ de combustibles fósiles en la generación de energía eléctrica para el año 2024, del $60 \%$ en el 2035 y del $50 \%$ en el 2050 (DOF, 2015).

De igual manera, referente a las emisiones contaminantes, en la Ley General de Cambio Climático se establece que México asume el objetivo de reducir al año 2020 un 30\% de emisiones con respecto a la línea de base; así como 50\% de emisiones al 2050 en relación con las emitidas en el 2000. No obstante, las acciones llevadas a cabo a nivel federal en cuestiones energéticas para la disminución de emisiones contaminantes y para la participación de energéticos renovables no parecieran estar encaminadas al cumplimiento de dichas metas (DOF, 2018).

En la actualidad, México depende principalmente de los combustibles fósiles para la generación de electricidad. Durante 2017 (cuadro 1), la producción de energía primaria en el país fue de un $90.6 \%$ proveniente de fuentes fósiles y energía nuclear, mientras que sólo el $9.5 \%$ provino de fuentes renovables, como geoenergía, solar, eólica, biomasa e hidroenergía (SENER, 2017).

Considerando que México es uno de los cinco países con mayor potencial solar a nivel global, pues recibe un promedio de energía solar de $5 \mathrm{kWh}$ por cada metro cuadrado al día (SE y ProMéxico, 2017), se ha planteado como un eje clave de acción el incremento de la incorporación de energías renovables en el actual mix energético del país por medio del uso de la tecnología fotovoltaica, principalmente, con proyectos a gran escala y la generación distribuida.

De acuerdo con lo definido por la Ley de la Industria Eléctrica, la generación distribuida es la generación de energía eléctrica realizada por un generador exento (con una capacidad instalada menor de $500 \mathrm{~kW}$ ); la central eléctrica se interconecta a un circuito de distribución que contenga una alta concentración de Centros de Carga. La interconexión de la central eléctrica deberá ser a la Red General de Distribución, que, en el caso de México, es la red de la Comisión Federal de Electricidad. 
Cuadro 1. Estructura de la producción de energía primaria en México en el año 2017

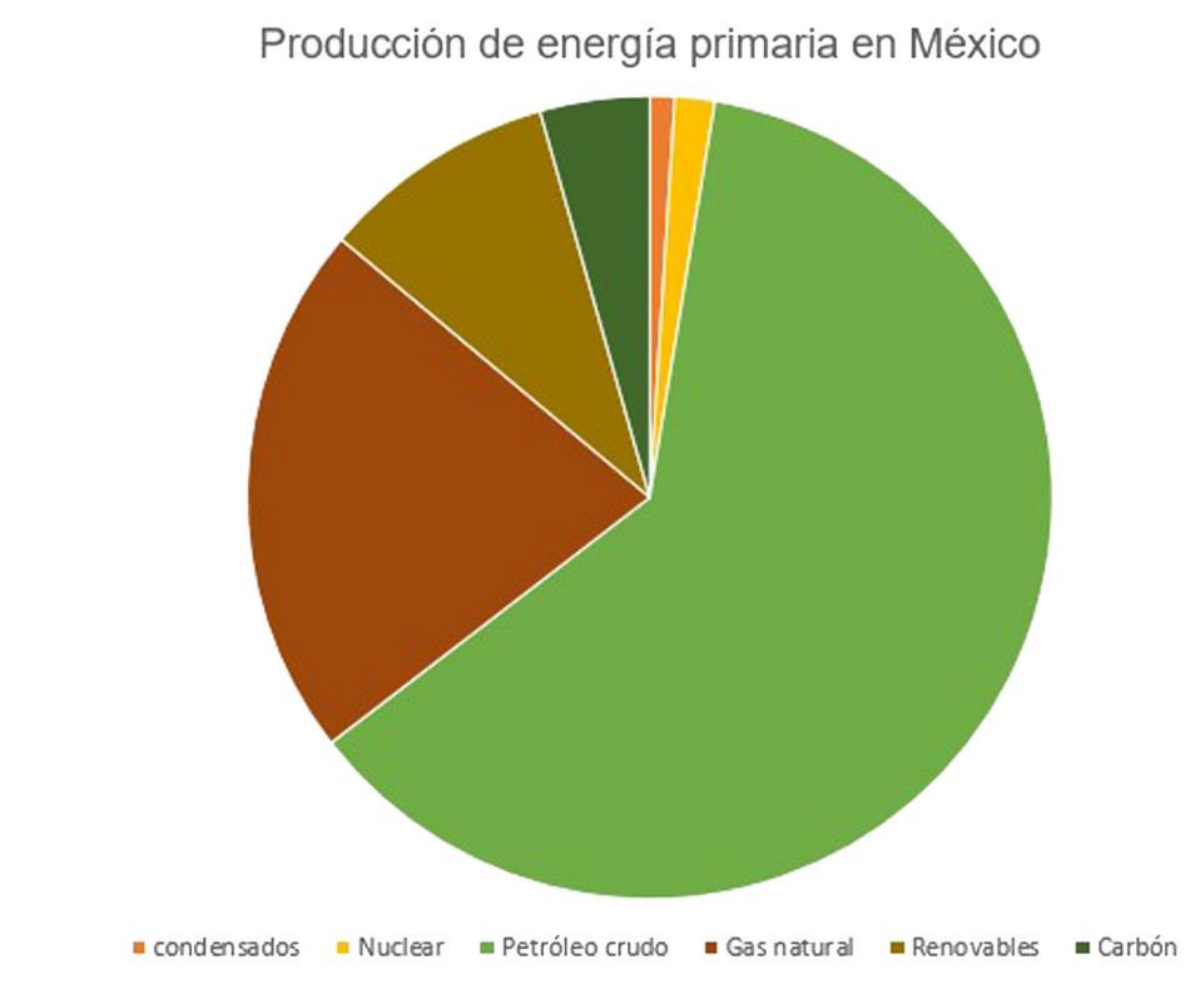

Fuente: SENER (2017), modificado por autores.

Asimismo, la vivienda ha sido un elemento importante en esta búsqueda por disminuir el uso de combustibles fósiles para la generación de electricidad, lo cual puede atribuirse a que en los sectores residencial, comercial y público la electricidad fue el energético de mayor consumo, con 34.3\% (SENER, 2017: 35). Para la International Electrotechnical Commission (2010), la electricidad es la forma de energía más fácil de controlar, transportar y distribuir; es la más limpia en el punto de uso respecto a otras fuentes de energía; por tanto, podría ser el factor que más contribuya a la mitigación del cambio climático (citado por CONUEE, 2017: 14).

Se ha puesto atención especial en incrementar el uso de ecotecnologías que funcionen con energía solar, como los calentadores solares y los paneles fotovoltaicos en la vivienda, interconectados a la red general de distribución correspondiente a la Comisión Federal de Electricidad, por medio de la generación distribuida.

No obstante, para algunos autores, como Fornillo (2017), no es tan adecuado recurrir a la generación distribuida si se busca hacer un cambio hacia un modelo descentralizado de energía eléctrica, pues "es preferible hablar de generación autónoma y comunitaria antes que distribuida, ya que este último adjetivo remite al supuesto de que la energía sería una, central y unificada; una imagen que debemos deconstruir" (Fornillo, 2017: 51). 
El sector vivienda en México ha buscado evolucionar hacia la sustentabilidad, para lo cual se ha desarrollado la Política Nacional de Vivienda, con el fin de promover el desarrollo ordenado y sustentable del sector, mejorar y regularizar la vivienda urbana, así como construir y mejorar la vivienda rural. Atiende la sustentabilidad desde tres pilares: el ambiental, alusivo al control de la mancha urbana y diseño eficiente de la vivienda; el económico, referente al aprovechamiento de la infraestructura urbana y equipamiento existente, así como al aumento de la eficiencia energética de las viviendas; y el social, con el cual se busca mejorar la calidad de vida con viviendas más confortables y mejor ubicadas (SEDATU y CONAVI, 2016).

Dentro de la Política Nacional de Vivienda, con la finalidad de impulsar un modelo de vivienda sustentable, se desarrolló la Política de Vivienda Sustentable, siendo precursor el programa Hipoteca Verde, que es el mecanismo con mayor trascendencia para el financiamiento de tecnologías eficientes en la vivienda. Su objetivo es equipar a las viviendas compradas, construidas, remodeladas o ampliadas con un crédito del INFONAVIT con ecotecnologías que ayuden a ahorrar agua, luz y gas, como llaves, inodoros, focos, aislantes térmicos, calentadores solares, refrigeradores y estufas eficientes, así como lavadoras ahorradoras grado ecológico, entre otros (INFONAVIT, 2020).

Al ser un programa a nivel federal existente en el país que ha tenido gran difusión, se esperaría que contribuya a aumentar el uso de energías renovables entre la población con la adquisición de tecnología fotovoltaica; sin embargo, se identifican algunas debilidades y cómo éstas podrían estar generando conflicto para alcanzar las metas de transición energética a tiempo.

\section{Metodología}

Con el objetivo de identificar los principales conflictos entre la política de vivienda sustentable en el país y las metas de transición energética, se realizó una investigación de tipo exploratoria, para llegar a una de tipo explicativa. La investigación exploratoria consiste en examinar un problema de área del conocimiento poco estudiado o sin estudiarse, y el investigador acude a la realidad para familiarizarse con ella (Maletta, 2009). En este caso, la transición energética en México desde la teoría y la academia ha sido un tema que se ha abordado poco, por lo cual existe un desconocimiento sobre la realidad que se vive en el país referente a las acciones, los programas y las metas de transición energética. Asimismo, las investigaciones exploratorias pueden ser una primera aproximación a un tema específico antes de tratarlo en un trabajo investigativo de más profundidad; es un proceso para obtener información básica sobre el problema de investigación. 
Por otro lado, las investigaciones explicativas pretenden responder las causas de eventos y fenómenos físicos o sociales presentes en la realidad estudiada. Este tipo puede ser la parte preliminar en un proceso de producción científica, donde se realizan actividades de estudio y exploración. Su objetivo principal es definir el por qué y para qué del objeto de estudio; además, ayudan a establecer relaciones causales entre variables que surgen de un modelo teórico. En este sentido, el identificar los principales conflictos a los que se enfrenta la transición energética en México en el área de vivienda puede servir como una etapa preliminar que permitirá desarrollar investigaciones de mayor profundidad.

De esta manera, se comienza con un análisis teórico del concepto de transición energética, así como de la evolución de la política de vivienda sustentable en México, para ello se recurre a la revisión documental de textos académicos, como artículos científicos, informes gubernamentales, leyes, normas y programas, lo cual permite identificar los conflictos principales de acuerdo con lo que se dice en la teoría y lo que se está haciendo en la práctica. Para finalizar, por medio del análisis e interpretación de la información recopilada, se expondrán aquellas premisas que resultarían fundamentales que el gobierno mexicano tomara en cuenta para llevar a cabo una transición energética.

\section{La transición energética}

La transición energética se entiende como "el cambio en la composición (estructura) del suministro de energía primaria, el cambio gradual de un patrón específico de provisión de energía a un nuevo estado de un sistema energético" (Smil, 2010: vii). Markard, Raven y Truffer (2012) sostienen que implica cambios de gran alcance en diferentes dimensiones: en lo tecnológico, lo material, lo organizativo, lo institucional, lo político, lo económico y lo sociocultural. Además, involucran una diversidad de actores $\mathrm{y}$, generalmente, se desarrollan en periodos prolongados (por ejemplo, 50 años o más).

Por su parte, para Fornillo (2017: 52), una transición no solamente "supone combatir las consecuencias negativas del cambio climático y aumentar los niveles de ahorro y eficiencia energética", sino que además involucra a la "innovación tecnológica local, a la transformación y actualización de la infraestructura energética que hace de base a cualquier país" (Fornillo, 2017: 52), y además requiere modificar el patrón energético, lo cual a su vez transformaría la idea de consumo actual, nuestra cultura y la idea de energía infinita.

En el ámbito académico, la discusión teórica sobre el tema de transición energética está enfocada hacia la búsqueda de un modelo alternativo al modelo tradicional de energía eléctrica, empleando términos como transición socioecológica (Bertinat y Chemes, 2018), transición socio-energética (Fornillo, 2017), transición energética justa (Bertinat, 2016) y transición energética sustentable (Aklin y Urpelainen, 2013); además, se han desarrollado movimientos como Postcarbon cities originado en Estados Unidos durante 2005, y el Transition towns nacido en Irlanda en 2006. 
No obstante, parece haber un consenso sobre las características principales que debiera tener una transición energética en la actualidad:

- Hacer la transición hacia un modelo eléctrico que sea descentralizado, donde no existan los oligopolios energéticos.

- Modelo eléctrico equitativo, con el cual se erradique la desigualdad energética.

- La transición deberá estar acompañada no únicamente de cambios referentes al tipo de energético empleado, la tecnología y la infraestructura, sino, además, con un cambio profundo en la idea cultural sobre el consumo de energía.

- Deberá haber comunidades más solidarias con sentido de cooperación.

- Deberán utilizarse fuentes energéticas renovables.

No obstante, los distintos movimientos y conceptos presentan diferencias marcadas entre sí. Postcarbon cities defiende la posibilidad de tener un crecimiento económico ilimitado que podrá ser alcanzado con el desarrollo de nuevas tecnologías y construcción de las infraestructuras adecuadas en los sectores energético y de transporte (Bermejo, 2013). Mientras que Transition towns apoya la idea de la necesidad de tener un descenso energético, pues la crisis energética obligará a crear sociedades austeras, porque las energías renovables no podrán suministrar una cantidad de energía semejante a la que aportan los combustibles fósiles (Bermejo, 2013).

Asimismo, los conceptos de transición socioecológica y transición socio-energética se han planteado desde una visión proveniente del sur global y muestran características más inclinadas a la búsqueda de la equidad en el acceso a la energía renovable y de la erradicación de la pobreza energética, así como también en resaltar la importancia de desarrollar valores de comunidad y cooperación en las comunidades. En cambio, los principales países impulsores de los movimientos Postcarbon cities y Transition towns se localizan en el norte global.

Por su parte, el concepto de transición energética justa ha sido adoptado desde el sur global, pero sus orígenes pueden verse desarrollados desde diferentes áreas de estudio, como lo mencionan Heffron y McCauley (2018): justicia energética (McCauley et al., 2013), justicia ambiental (Capek, 1993; Walker \& Buckeley, 2006), y justicia climática. Asimismo, cada una de estas áreas maneja diferentes perspectivas:

- La justicia climática se refiere a compartir los beneficios y las cargas del cambio climático desde un enfoque de derechos humanos.

- La justicia energética se refiere a la aplicación de los derechos humanos en todo el ciclo de vida de la energía (desde la cuna hasta la tumba).

- La justicia ambiental tiene como objetivo tratar a todos los ciudadanos por igual e involucrarlos en el desarrollo, la implementación y la aplicación de leyes, reglamentos y políticas ambientales (Heffron y McCauley, 2018). 
En este sentido, parecieran existir múltiples posibles caminos de acción a seguir para llevar a cabo una transición energética; sin embargo, es importante considerar las características propias de la región, la sociedad, de infraestructura, capacidades tecnológicas, las metas a alcanzar, etcétera.

En México, de acuerdo con lo expuesto en la Ley de Transición Energética, la transición enfoca sus acciones en buscar el incremento gradual de la participación de las Energías Limpias en la Industria Eléctrica con la finalidad de cumplir con las metas establecidas en materia de generación de energías limpias y de reducción de emisiones; no obstante, resalta la falta de consideración por conceptos como equidad, solidaridad, cooperación y justicia, así como la participación activa de los ciudadanos en el proceso.

Asimismo, en la Estrategia Nacional de Cambio Climático (CONUEE, 2017), las líneas de acción planteadas para acelerar la transición energética hacia fuentes de energía limpia sobresalen las acciones para aumentar la producción energética, ya sea con mejoras en la red, la conexión, la tecnología y la participación con proyectos a gran escala y por medio de la generación distribuida.

En el actual periodo de gobierno 2019-2024, se habla de rescatar el sector energético en el país por medio de Petróleos Mexicanos (PEMEX) y de la Comisión Federal de Electricidad (CFE) para que vuelvan a operar como palancas del desarrollo nacional, rehabilitando las refinerías existentes, construyendo nuevas y modernizando las hidroeléctricas. Por otro lado, se propone impulsar el desarrollo sostenible del sector e incorporar poblaciones y comunidades a la producción de energía con fuentes renovables, a fin de dotar de electricidad a las pequeñas comunidades aisladas. En este sentido, la transición energética en México no pareciera estar enfocada en seguir alguno de los caminos propuestos desde el ámbito académico y la teoría.

\section{Política de vivienda sustentable. Programa Hipoteca Verde}

Las políticas tienen como objetivo principal mejorar las condiciones de vida de la sociedad y pueden ser diseñadas para regular diversos aspectos, como educación, salud, vivienda, comunicación y transporte, entre otros. En el caso de las políticas para la vivienda, si bien la preocupación por parte del gobierno sobre cuestiones habitacionales en México se data desde 1915 aproximadamente, fue hasta 1996 cuando empezó a relacionarse con temas afines a la eficiencia energética y se expidieron las primeras Normas Oficiales Mexicanas (NOM) para la eficiencia energética en equipos del sector residencial, con aplicación a refrigeradores y equipos de aire acondicionado de ventana.

Un año después se creó el Fideicomiso para el Ahorro de Energía Eléctrica (FIDE), el cual continuó el esfuerzo de la iniciativa Ilumex; estaba orientado al cambio de lámparas eficientes en las viviendas. Estos fueron los primeros esfuerzos por parte del gobierno para introducir tecnologías más eficientes a la vivienda (CAPSUS, 2017). 
No obstante, en 2001, se inició el proceso de transición hacia una política de vivienda para fomentar la sustentabilidad y se creó la Comisión Nacional de Fomento a la Vivienda (CONAFOVI), hoy Comisión Nacional de Vivienda (CONAVI); La Ley de Vivienda le asignó supervisar que las acciones de vivienda se realicen en torno al cuidado del desarrollo urbano, el ordenamiento territorial y el desarrollo sostenible (CAPSUS, 2017).

Durante el 2007, se crearon programas enfocados a mejorar la sustentabilidad de la vivienda en México; como consecuencia, se creó el Programa Nacional de Vivienda, a partir del cual el Instituto del Fondo Nacional de la Vivienda para los Trabajadores (INFONAVIT) desarrolló Vivir INFONAVIT, diseñado a partir de tres ejes: entorno, vivienda y comunidad. Así, desde el 2008, se vislumbró el Programa Hipoteca Verde, basado en financiar la adquisición de ecotecnologías para las viviendas de los derechohabientes (CAPSUS, 2017: 12). Sin embargo, se implementó de forma obligatoria hasta el 2011.

Asimismo, con la mejora constante del programa Hipoteca Verde, en 2011, a través de los objetivos del Programa de Energía Sustentable en México de la GIZ, el INFONAVIT, el consorcio Gopa-Integration (por encargo de GIZ) y la Fundación IDEA (con fondos de la Embajada Británica), se desarrolló el programa Sisevive-Ecocasa (CAPSUS, 2017), el cual permite conocer el nivel de eficiencia de la vivienda de acuerdo con su consumo proyectado de energía y agua; para ello, utiliza dos herramientas de cálculo: SAAVi (Simulador de Ahorro de Agua en la Vivienda) y DEEVi (herramienta para el Diseño Energéticamente Eficiente).

En 2012, entra en vigor la Ley General de Cambio Climático, a través de la cual México adquiere el compromiso de reducir las emisiones de gases de efecto invernadero (GEI) y evitar la deforestación. Para 2013, el programa Hipoteca Verde expandió sus beneficios a los acreditados con un ingreso salarial de 1.6 veces salario mínimo mensual y se incorporaron los focos LED en los criterios del programa. En 2014 se desarrolló la Acción de Mitigación Nacionalmente Apropiada (NAMA por sus siglas en inglés) de vivienda existente, y en lo referente a Hipoteca Verde se abrió la convocatoria para la incorporación de proveedores de productos autorizados.

En 2015 se agregaron varias tecnologías, como electrodomésticos, y se incorporó el 100\% de lámparas LED, así como medidas para reducir la ganancia de calor en climas cálidos. En 2016, se publicaron modificaciones a la NOM 020 sobre la eficiencia energética en edificaciones. 
Durante 2017, la Secretaría de Desarrollo Agrario, Territorial y Urbano (SEDATU), la CONAVI, la Secretaría de Energía (SENER), la Nacional Financiera (NAFIN), la Comisión Federal de Electricidad (CFE) y el FIDE implementaron el Programa de Mejoramiento Integral Sustentable para Vivienda Existente, ofreciendo financiamiento a las familias para tener acceso a ecotecnologías para su vivienda. Comenzó de manera piloto en abril del 2017 en Campeche, Chihuahua, Cuernavaca, Guadalajara, Mérida, Monterrey, Puebla, Pachuca, Toluca y Veracruz; no obstante, en julio del mismo año ya participaban veinticuatro ciudades (Ávila y Toledo, 2017).

En 2018, con el fin de fortalecer el programa Hipoteca Verde, se le realizaron modificaciones importantes, como el cumplimiento obligatorio de una línea base de eficiencia energética y ambiental, incluida en el precio de venta de la vivienda; asimismo, el monto de Hipoteca Verde se convirtió en opcional.

De esta breve revisión, se observa que la evolución del programa Hipoteca Verde en los últimos diez años no ha presentado cambios sustanciales respecto al tipo de ecotecnologías ofrecidas, la manera de adquirirlas y las medidas para brindar resultados significativos. Si bien este programa emplea el término ecotecnologías para una amplia gama de electrodomésticos y equipamiento para la vivienda, pareciera enfocarse en el posible ahorro económico que éstos suponen, en lugar de buscar aprovechar los recursos naturales y producir un impacto mínimo en el medio ambiente.

El principal programa existente a nivel federal, Hipoteca Verde, pareciera facilitar la adquisición de equipamientos, como aires acondicionados, lavadoras, estufas, refrigeradores, etcétera, ahorradores, que continúan funcionando por medio de energías obtenidas de la quema de combustibles fósiles, en lugar de dar mayor énfasis en la promoción de conjunto de técnicas que funcionen a base de energías renovables.

Este problema se observa en regiones de clima cálido, donde el gasto energético en la vivienda se eleva por el uso de aires acondicionados y, de acuerdo con la CONUEE, en los últimos cuatro años la demanda eléctrica por confort térmico ha crecido más del 50\% en zonas de clima cálido bajo tarifas 1B y 1C (SENER y CONUEE, 2018). Mientras INFONAVIT considere al aire acondicionado como una ecotecnología de máximo ahorro es difícil que los productos como paneles y calentadores solares compitan con un equipamiento arraigado a la estructura social de los ciudadanos, que brinda beneficios inmediatos a su calidad de vida por medio de la satisfacción de sus necesidades de confort térmico.

Además, el programa Hipoteca Verde, de 2008 a 2018, no ha presentado resultados significativos que ayuden a disminuir el problema de demanda eléctrica en regiones de clima cálido por el uso de equipos de enfriamiento. En este sentido, es importante preguntarse si este programa realmente está contribuyendo de la manera más adecuada a incorporar ecotecnologías que generen un mínimo impacto en el medio ambiente, y podría generarse la interrogante ¿qué necesita el programa Hipoteca Verde para convertirse en un actor clave en el sector residencial que contribuya a lograr las metas establecidas? 
De acuerdo con Acosta y Aguilar (2018), el programa Hipoteca Verde puede ser considerado como una interesante propuesta por parte del gobierno como aporte ante el problema global de mitigación del cambio climático, sin embargo, no se ha planteado adecuadamente ni replanteado lo suficiente para hacer salir a la política de su área de confort. En este sentido, a continuación se exponen tres conflictos principales de la política de vivienda sustentable con las metas de transición energética en el país.

\section{Principales conflictos}

\section{Desigual acceso a la energía renovable}

Contrario a lo que se pudiera pensar, los sectores más pobres de la población gastan más en energía, y la calidad de ésta es menor. De acuerdo con la OIEA (2018), el tener disponibilidad y acceso a la energía repercute directamente en la pobreza, las oportunidades de empleo, la educación, la transición demográfica, la contaminación en ambientes cerrados y la salud. Las personas con mayores recursos económicos disponen con mayor facilidad a energía para la iluminación, la calefacción y la preparación de comida; además, la energía es más limpia, segura, fiable y asequible. En cambio, las personas con recursos más bajos no suelen tener acceso tan fácil a los diferentes energéticos que requieren para alimentarse, calentarse o refrigerarse.

Por lo tanto, es importante considerar las condiciones del lugar de destino de la energía, es decir, la vivienda; así como la estructura del proceso que hace posible la llegada de la energía eléctrica a las viviendas. Existe el denominado modelo tradicional del sistema eléctrico, encargado de la generación, transmisión y distribución de electricidad; no obstante, puede tener un papel clave en la contribución de la desigualdad energética en las ciudades. El elevado costo de las ecotecnologías propicia la desigualdad de acceso a fuentes alternativas de energía, como la solar o la eólica. Se concibe que ser un ciudadano preocupado por el medio ambiente es un lujo y que los pobres son demasiado pobres para ser ecologistas (Martinez-Alier, Temper, Demaria, 2014: 53). Sin embargo, es posible aplicar otras medidas para la incorporación de energías renovables en el sistema eléctrico.

2. No se hace énfasis en la necesidad de disminuir el consumo de energía eléctrica en los hogares

De acuerdo con Fornillo (2017), para lograr una transición energética, debemos convertirnos en una sociedad desmaterializada que modifique sus parámetros de consumo. Por su parte, Bertinat y Chemes (2018) sostienen que para el desarrollo de alternativas y la construcción de transiciones es preciso aceptar las limitaciones físicas existentes y la imposibilidad del crecimiento ilimitado en un planeta con recursos finitos. Mientras que movimientos como Transition Towns parten de la idea de que la crisis energética obligará a crear sociedades muy austeras, porque niega que las energías renovables puedan suministrar una cantidad de energía equivalente a las energías convencionales (Bermejo, 2013: 222). 
En este sentido, se considera un punto importante la disminución de la energía que es consumida para lograr la transición energética esperada, y para esto no sólo se requiere tener casas o electrodomésticos más eficientes energéticamente, sino que es necesario modificar nuestra idea de consumo y la relación que tenemos con la energía. Como menciona Bertinat (2016), "esto plantea la necesidad ineludible de trabajar en torno a los procesos de construcción del deseo, referido a disputar la cultura dominante, que plantea la necesidad de tener cada vez más bienes materiales y energía para alcanzar la felicidad" (Bertinat, 2016: 08).

No obstante, la política de vivienda sustentable en México, en especial el programa Hipoteca Verde, no sólo refuerza la idea de mercantilización de la energía, sino que además promueve mantener el estilo de vida de consumo desmedido de energía. Los equipamientos a los cuales pueden accederse por medio del crédito Hipoteca Verde no son sólo ecotecnologías; es decir, el programa facilita la adquisición de equipamientos ahorradores, como aires acondicionados, lavadoras, estufas, refrigeradores, entre otros, los cuales continúan funcionando por medio de energías obtenidas de la quema de combustibles fósiles, en lugar de dar mayor énfasis en la promoción de conjunto tecnologías que trabajen a base de energías renovables.

Por lo tanto, mientras INFONAVIT siga considerando electrodomésticos ahorradores de uso diario es difícil que los productos como paneles y calentadores solares compitan con un equipamiento arraigado a la estructura social de los ciudadanos, que brinda beneficios inmediatos a la calidad de vida de las personas por medio de satisfacer sus necesidades básicas.

De acuerdo con la Secretaría de Energía (SENER), el consumo de energía en México va en aumento cada año, presentándose un consumo de energía per cápita con un aumento de $6.1 \%$ en 2016 con respecto al año 2015 (SENER, 2016). Mientras que a nivel global el aumento en la demanda de energía eléctrica se encuentra constante (cuadro 2), durante el 2018 aumentó en un 2.3\%, lo cual a su vez originó que las emisiones globales de CO2 relacionadas con la energía aumentaran un 1.7\%, presentando un máximo histórico de 33.1 gigatonelada de CO2 (IEA, 2019). 
Cuadro 2. Consumo de electricidad per cápita en el mundo 1990-2016

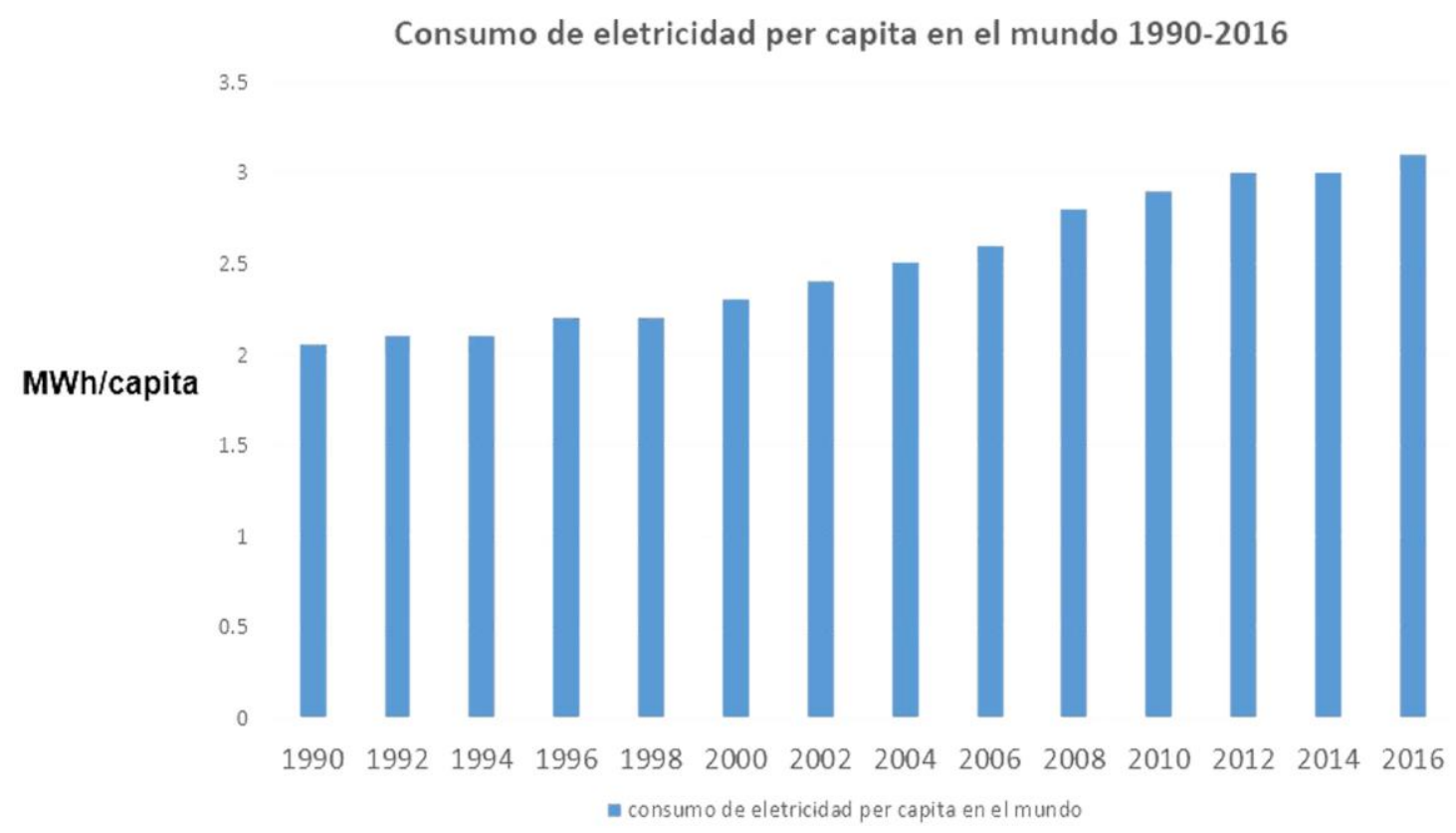

Fuente: IEA (2019).

\section{La generación distribuida podría no encaminar hacia un modelo descentralizado}

Este último conflicto podría resultar controversial, pues la generación distribuida ha llegado a ser la principal forma de impulso para el uso de energía renovable en la vivienda. Por otro lado, son innegables los aspectos positivos que supone como estrategia, pero es importante no dejar de lado que puede caer fácilmente en un proceso de mercantilización e inequidad, así como entender que por medio de la generación distribuida se continúa dependiendo de una red principal de distribución.

Se dice que el actual modelo energético existente en el país se caracteriza por ser poco equitativo, muy mercantilizado, poco democrático, extractivista y que está pensado para enriquecer a unos cuantos por medio de los oligopolios energéticos y no para mejorar la calidad de vida de las personas (Bertinat, 2016). En este sentido, con los crecientes contrapuntos teóricos surgidos en los últimos años sobre los cambios que necesita el modelo tradicional de energía eléctrica enfocados a un modelo descentralizado, desmercantilizado, equitativo y democrático, se ha promovido la generación distribuida a pequeña y a mediana escalas en los lugares de consumo final; no obstante, aún dependen de la red principal del sistema eléctrico pues están conectados a ésta, lo cual recae en la misma idea de centralidad.

Por consiguiente, la generación distribuida remite al supuesto de que la energía sería una, central y unificada; una imagen que debemos deconstruir si queremos lograr una transición energética justa (cuadro 3). 


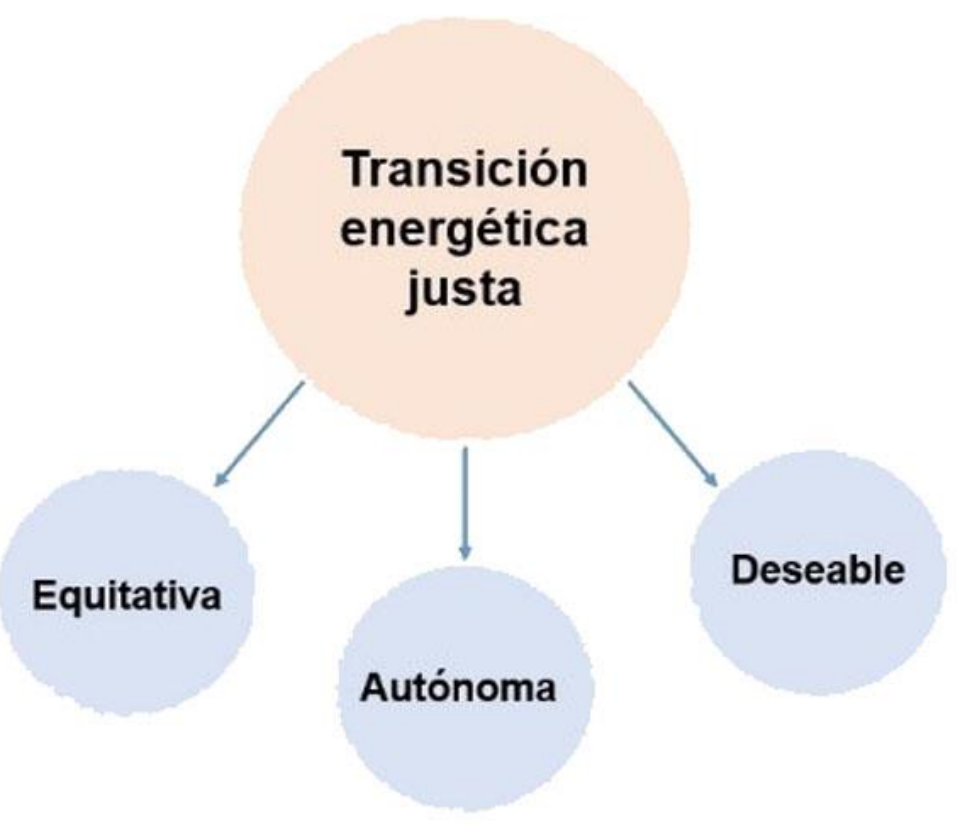

Fuente: elaboración propia.

\section{Discusión}

Los tres conflictos mencionados se relacionan con el acceso a la energía renovable, el papel directo que ejerce la sociedad en el proceso de incorporación de energías renovables y lo obsoleto del sistema eléctrico nacional. En este sentido, México podría basarse en una serie de premisas fundamentales que ayudarían a disminuir estos problemas y permitirían encaminar la transición energética hacia el cumplimiento de las metas establecidas; a saber:

- Impulsar y fomentar una conciencia ambiental (cuadro 4) en los ciudadanos, la cual se aplique a todos los aspectos y haga énfasis en los estilos de habitar sus viviendas y los consumos energéticos en sus hogares, apoyando la idea de crear sociedades más austeras y más consientes. Para esto, se requiere que la sociedad sea incluida en el proceso que conlleva la generación, transmisión y distribución de energía eléctrica, ya sea participando directamente en la generación de energía o formado parte de la toma de decisiones. 
Cuadro 4. Dimensiones de la conciencia ambiental

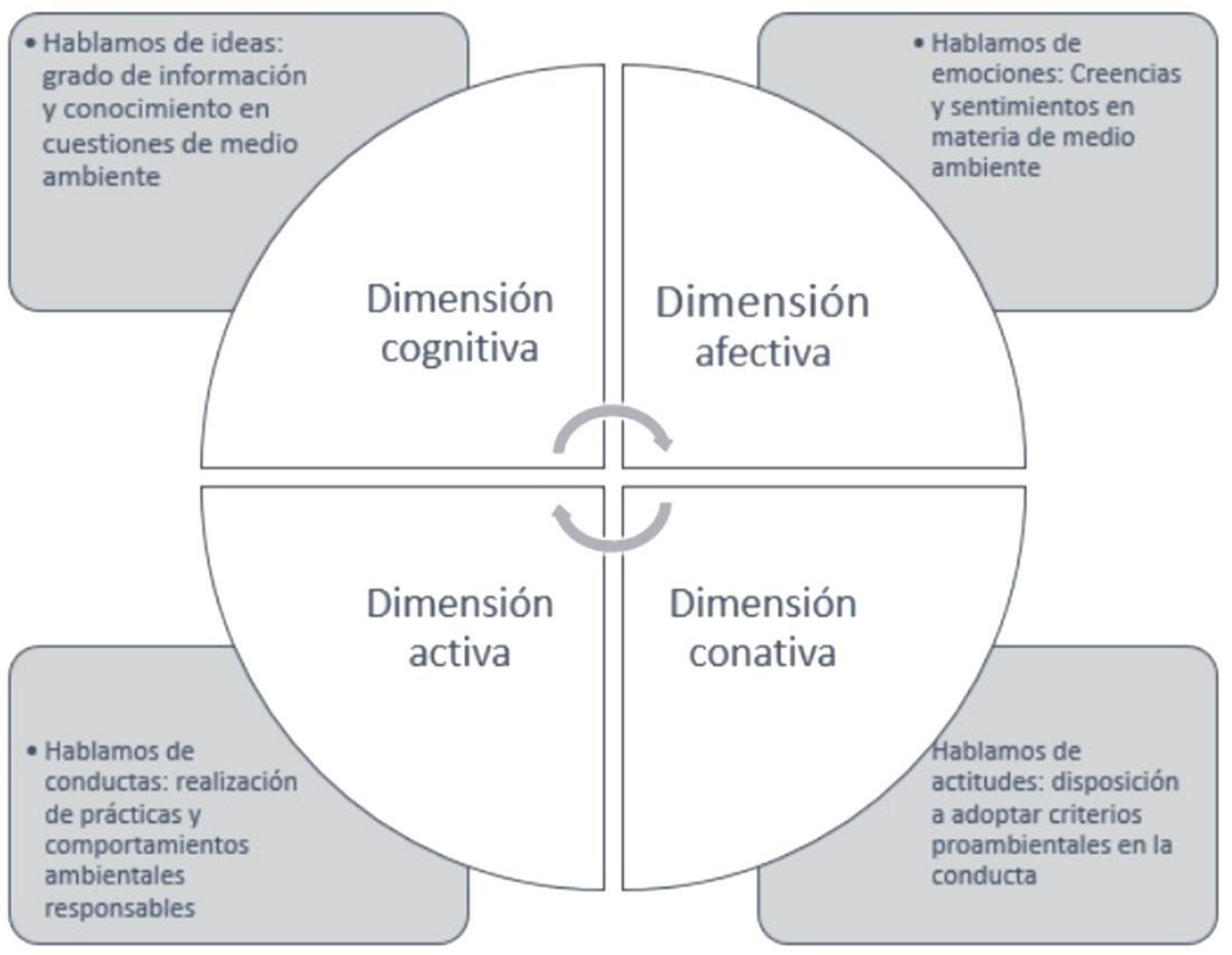

Fuente: Medina Lagrange (2018).

- Apegarse a la idea de una transición energética justa teniendo como punto rector la justicia. El trabajo en conjunto con las instituciones para la creación de planes y estrategias fortalecería las oportunidades de lograr acercarse a las metas de transición energética. Además, al tener presente a quién está dirigida la transición posibilitará establecer un proceso justo y deseable. Por lo tanto, en lugar de pensar en transiciones energéticas con énfasis en lo social, con un enfoque hacia un mayor desarrollo económico, o preocupadas únicamente por lo ecológico, se deberá pensar en transiciones justas, con todo lo que esto signifique; es decir, justas para la sociedad, para la ecología, para los organismos encargados, adaptadas a las características del sitio, pero sobre todo sustentables.

- Plantear una transición energética requiere de un cambio radical, lo cual significaría que no se debe buscar únicamente modificar la política de vivienda sustentable en México, sino hacer una transformación desde los cimientos del sistema eléctrico nacional, así como del sector de la construcción. 
Como ha sido mencionado, uno de los programas principales desarrollados por la política de vivienda sustentable en el país, el programa Hipoteca Verde, tiene un enfoque basado principalmente en el aspecto económico de los derechohabientes; en México, el mayor problema de la vivienda social es de aspecto económico, ocasionando que la ecoinnovación y el grado de implementación de eco-tecnologías se resuma en la capacidad de financiamiento.

Por lo tanto, además de modificar los procesos de producción y aprovechamiento de la materia prima en la industria eléctrica y la forma de distribución de la electricidad hacia los ciudadanos, se requiere construir de forma sustentable. De acuerdo con Paz, Rivera y Ledezma (2016: 55), la vivienda sustentable "a la vez de cumplir con las condiciones a favor de la huella ecológica debe llenar los aspectos social y económico", lo cual implica que, además de preocuparse por solventar los aspectos tecnológicos como la eficiencia energética en la vivienda, deben ser considerados los aspectos humanos, como la viabilidad social y económica de las viviendas, teniendo en cuenta siempre hacia quién va dirigida.

Por lo tanto, para que la vivienda en México sea realmente sustentable, "se requiere diseñar modelos de sistemas urbanos y arquitectónicos de alta eficiencia, basados en determinación de estructuras físicas, ambientales y sociales sustentables, a partir de factores y elementos que garanticen calidad de vida en la habitabilidad de las ciudades y la vivienda" (Ávila, 2019: 96).

- Apegarse a las capacidades de cada región del país para la producción de energía eléctrica por medio de fuentes renovables, así como a las capacidades y necesidades de cada ciudad; buscar en lo posible que la energía sea producida localmente.

- Las ciudades pueden llegar a ser un punto central en las respuestas hacia el combate del cambio climático. De acuerdo con Bulkeley, Castan \& Edwards (2012), a finales de los años 80 y principios de los años 90, pequeñas ciudades y grupos de municipalidades empezaron a incluir el cambio climático en sus agendas; se enfocaban principalmente a reducir las emisiones de gases de efecto invernadero; argumentaban que las ciudades se convirtieron en parte esencial de la respuesta al cambio climático debido a su densidad de actividades, a los productores de estos gases y al rol potencial de las autoridades municipales en sus procesos de regulación.

Asimismo, un urbanismo bajo en carbón o low carbon a escala ciudad podría estar enfocado en "el desarrollo de estrategias como formas de infraestructura energética 'autosuficientes' y de bajas emisiones de carbono, incluida la generación descentralizada, proyectos Smart grid y desarrollos de cero carbón" (Bulkeley, Castan \& Edwards, 2012: 549). La importancia recae en cambiar la escala y pasar de lo nacional a lo local. 
De acuerdo con North y Longhurst (2013), iniciativas de transiciones generadas localmente que interactúen con las estructuras locales de poder podrían promover la transición hacia economías bajas en carbón de manera más efectiva. Además, el metabolismo de las grandes ciudades es insostenible, pues se abastece de redes mundiales; se dota de energía a través de centrales eléctricas de combustibles fósiles conectadas a una red y enviando sus desechos a otros lugares (Steel, 2008, citado por North y Longhurst, 2013). Sobre esta idea de abastecimiento de energía eléctrica por medio de conexión a una gran red se desarrolla la siguiente premisa, tomando en cuenta la descentralización del modelo del sistema eléctrico nacional:

- Transitar del modelo tradicional del sistema eléctrico que se tiene en el país hacia un modelo descentralizado; este cambio requiere la transformación de la infraestructura, del marco regulatorio y de la mentalidad de la sociedad. Modernizar a la Comisión Federal de Electricidad (CFE) para que su estructura y organización se adapte a los nuevos requerimientos del sistema eléctrico nacional. Promover el manejo del servicio de energía eléctrica a nivel municipal o regional de manera descentralizada.

Como caso de éxito, cabe mencionar a las comercializadoras de energía eléctrica pública. Ejemplo de esta estructura de sistema eléctrico es el Ayuntamiento de Barcelona, el cual, como de uno de sus ejes estratégicos de actuación, ha desarrollado su propia empresa de energía pública con la finalidad de recuperar la soberanía energética y como contraparte a los oligopolios energéticos y a la privatización energética.

Barcelona Energía (BE) es una compañía distribuidora de energía; es propiedad del ayuntamiento y se abastece de energía limpia (renovable), producida localmente; busca abonar a la idea de la energía como un derecho básico y un servicio público garantizado. Asimismo, este modelo de sistema eléctrico propone ser distribuido, participativo y democrático.

Por otro lado, el modelo energético desarrollado por el ayuntamiento de Barcelona promueve reducir el consumo energético y hacer uso racional de la energía por medio de devolver el protagonismo de la transformación a los ciudadanos, incrementando la cultura energética de los ciudadanos y capacitándolos para participar en este futuro sostenible (Barcelona Energía, 2020). 
Otro caso es el de "stadtwerke" o servicios públicos municipales establecidos en Alemania. Este modelo se encarga de manejar áreas diversas, como el agua potable o la energía eléctrica a través de los gobiernos locales; es decir, tienen compañías energéticas; se trata de descentralizar presentándose como una contraparte a las grandes corporaciones privadas de energía eléctrica.

Esta forma de manejar los servicios, como la energía eléctrica, apoya la economía local y regional; además, asegura un suministro de energía sustentable. De acuerdo con Wagner y Berlo (2017), ofrece oportunidades en el desarrollo de la eficiencia energética y las energías renovables, lo cual permite a las municipalidades dar servicios de energía de alta calidad que tendrían beneficio para las generaciones presentes y futuras.

\section{Conclusiones}

El término transición energética ha cobrado fuerza en los últimos años, pues se encuentra en la agenda política de la mayoría de los gobiernos. No obstante, no existe una sola transición energética, sino una gran diversidad de caminos. En México, a la vivienda se le ha otorgado un papel clave en la búsqueda de estrategias para alcanzar las metas de energía y emisiones planteadas. Sin embargo, el actual modelo energético existente en el país, considerado el tradicional, el cual abarca territorios extensos, se basa en los combustibles fósiles; además, debido a la presencia de oligopolios o monopolios energéticos, no promueve una transición energética justa, equitativa, cooperativa y descentralizada.

$\mathrm{Al}$ analizar la política de vivienda sustentable en México, es posible observar que el programa de mayor cobertura a nivel nacional presenta algunos conflictos que impedirían alcanzar las metas energéticas a tiempo y empatizar con el debate teórico actual. Por lo tanto, se exponen tres propuestas para reforzar la política de vivienda sustentable en México.

En primera instancia, resulta de suma importancia considerar si el término ecotecnologías está siendo empleado adecuadamente dentro del programa Hipoteca Verde, ya que, si bien los proveedores autorizados contemplan adquirir por medio del monto del programa tecnologías que aprovechen energías renovables como la energía solar, éstas no forman parte de la línea base y son de las menos adquiridas en regiones donde el gasto energético en la vivienda es más elevado. Mientras se continúe ofreciendo a los electrodomésticos como ecotecnologías convencionales, se dificultará lograr el objetivo de aumentar la participación de energía renovable en el mix energético.

En segundo lugar, se requiere trabajar en un programa que promueva la educación ambiental. Fortalecer la conciencia ambiental en los ciudadanos es de gran importancia para disminuir el consumo de energía en los hogares; es imperante adquirir prácticas que permitan realmente promover la sustentabilidad en la vivienda. 
Por último, es fundamental ver más allá de la generación distribuida y considerar las diferentes estrategias que permiten tener un modelo energético realmente descentralizado. Si bien representan un reto mayor a nivel de infraestructura, de tecnología, de políticas y socialmente, podrían llegar a ser el camino necesario para lograr las metas planteadas, pues como menciona Fornillo (2017: 52): "modificar el patrón energético supone una modificación radical de nuestra idea de consumo, de nuestra experiencia y cultura atada al fetichismo del petróleo, es decir, a la idea de energía infinita, para recrear de manera radical nuestra vinculación con la naturaleza, en una común biosfera".

Por consiguiente, el programa Hipoteca Verde si bien ha sido un intento por mejorar la sustentabilidad de la vivienda en México, también es un intento demasiado moderado y discreto con múltiples deficiencias que deberían ser replanteadas apoyándose en el ámbito académico.

Asimismo, tener una política de vivienda sustentable en México que contribuya a alcanzar las metas de transición energética podría ser una tarea difícil si no se considera un cambio importante en el modelo del sistema eléctrico nacional que suministra de energía a las viviendas. Por lo tanto, como punto de partida, es necesario trabajar en las premisas fundamentales de una transición energética para el país.

\section{Referencias}

Acosta Rendón, J., y Aguilar Torres, G. (2018). El programa Hipoteca Verde del INFONAVIT ¿hacia una política de vivienda sustentable?, Vivienda y Comunidades Sustentables, (3), 25-34. Recuperado de: http://www.revistavivienda.cuaad.udg.mx/index.php/rv/article/view/36/55

Aklin, M. \& Urpelainen, J. (2013). Political Competition, Path Dependence, and the Strategy of Sustainable Energy Transitions, American Journal of Political Science, 57 (3), 643-658

Ávila García, E. y Toledo Rojas, J.E. (2017). Programa de mejoramiento integral sustentable en vivienda existente, Revista Eficiencia Energética, (15), pp. 05-09.

Ávila Ramirez, D. C. (2019). Implicaciones del metabolismo urbano en el cambio climático, Vivienda y $\begin{array}{llll}\text { Comunidades } & \text { Sustentables, } & 3 & \text { 79), }\end{array}$ http://www.revistavivienda.cuaad.udg.mx/index.php/rv/article/view/104/109

Barcelona Energía (2020). La comercializadora pública de energía. Recuperado de https:/ / www.barcelonaenergia.cat/es/la-comercializadora-de-energia-publica/

Bermejo, R. (2013). Ciudades postcarbono y transición energética. Economía Crítica, (16), 215-243.

Bertinat, P. (2016). Transición energética justa. Pensando la democratización energética. Análisis, (1), 1-20.

Bertinat, P. y Chemes, J. (2018) Aportes del sector energético a una transición socioecológica. V Cumbre Cooperativa de las Américas, documento de discusión.

Bulkeley, H., Castan Broto, V. \& Edwards, G. (2012) ‘Bringing ClimateChange to the City: Towards Low Carbon Urbanism?' Local Environment: The International Journal of Justice and Sustainability, 17(5), 545-551

Capek, S. (1993). The “Environmental Justice” frame: a conceptual discussion and an application, Social Problems, $40(1), 5-24$. 
CAPSUS (2017). Vinculación de Hipoteca Verde del INFONAVIT con el Sistema de Evaluación de la Vivienda Verde (Sisevive- Ecocasa). México. Recuperado de: http://portal.ruv.org.mx/wpcontent/uploads/2019/08/VVINC-Sisevive-Ecocasa2017.pdf

CONUEE (2017). Estrategia de transición para promover el uso de tecnologías y combustibles más limpios 2016. Secretaría de Energía, México.

DOF (Diario Oficial de la Federación) (2015). Ley para el Aprovechamiento de Energías Renovables y el Financiamiento de la Transición Energética. Recuperado de http://www.diputados.gob.mx/LeyesBiblio/abro/laerfte/LAERFTE_abro.pdf

DOF (Diario Oficial de la Federación) (2018). Ley General de Cambio Climático. Recuperado de http://www.diputados.gob.mx/LeyesBiblio/pdf/LGCC_130718.pdf

Fornillo, B. (2017). Hacia una definición de transición energética para sudamerica: Antropoceno, geopolítica y desarrollo, Revista Practicad de Oficio, 2 (20), 46-53. Recuperado de: https://static.ides.org.ar/archivo/www/2012/04/5-FORNILLO.pdf

Heffron, R. J., \& McCauley, D. (2018). What is the "Just Transition"?, Geoforum, 88, 74-77

INFONAVIT (2020). Hipoteca Verde. Recuperado de https://portalmx.infonavit.org.mx/wps/portal/infonavit.web/proveedores-externos/para-tugestion/desarrolladores/hipoteca-verde

IEA (2019). Global Energy \& $\mathrm{CO}_{2}$ status report. The latest trends in energy and emissions in 2018. International Energy Agency

Maletta, H. (2009). Epistemología aplicada: Metodología y técnica de la producción científica. Lima, Perú: Universidad Del Pacífico.

Markard. J, Raven, R. \& Truffer B. (2012). Sustainability transitions: An emerging field of research and it prospects. Research Policy, (41), pp. 955-067

Martinez-Alier, J., Temper, L. \& Demaria, F. (2014). Social metabolism and environmental conflicts in india, Indi@logs, vol. 1, pp. 51-83

Medina Lagrange, O. (2018). Estrategias de proyectos que fomentan la conciencia medioambiental y el bien común: Intervenciones de la arquitectura en el paisaje. Cuaderno de pedagogía universitaria, (15), pp. 3754

McCauley, D., Heffron, R.J., Stephan, H. \& Jenkins, K. (2013). Advancing Energy Justice: The triumvirate of tenets, International Energy Law Review, 32(3), 107-110.

North, P., \& Longhurst, N. (2013). Grassroots Localisation? The Scalar Potential of and Limits of the "Transition" Approach to Climate Change and Resource Constraint. Urban Studies, 50(7), 1423-1438.

OIEA (2018). Indicadores energéticos del desarrollo sostenible: directrices y metodologías. Austria

Paz Pérez, C.A., Rivera Herrera, N.L. y Ledezma Elizondo, M.T. (2016). El impacto de la sustentabilidad en la vivienda en serie de Nuevo León, Revista Contexto, 9 (11), 43-57. http://contexto.uanl.mx/index.php/contexto/article/view/87/82

SEDATU y CONAVI (2016). Vivienda sustentable en México. La NAMA como parte de la transformación del sector vivienda. México.

SENER (2016). Evaluación Rápida del Uso de la Energía, México

SENER (2017). Balance nacional de energía 2017. Recuperado de https://www.gob.mx/cms/uploads/attachment/file/414843/Balance_Nacional_de_Energ_a_2017.p $\mathrm{df}$

SENER y CONUEE (2018). Energía y edificaciones en México: Importancia y políticas públicas presentes y futuras (Balance nacional de energía 2017), México. 
Catalina Borbolla-Gaxiola y David Carlos Ávila-Ramírez: Principales conflictos entre la política de vivienda...

SE y ProMéxico (2017). La industria solar fotovoltaica y fototérmica en México. Recuperado de https://www.promexico.mx/documentos/biblioteca/industria-solar.pdf

Smil, V. (2010). Energy transitions. History, requirements, prospects. Estados Unidos: Praeger.

Wagner, O. \& Berlo, K. (2017). Remunicipalisation and Foundation of Municipal Utilities in theGerman Energy Sector: Details about Newly Established Enterprises, Journal of Sustainable Development of Energy, Water and Environment Systems, 5(3), 396-407.

Walker, G. \& Buckeley, H. (2006). Geographies of environmental justice, Geoforum, 37(5), 655-659. 